\title{
Identifying small groups of foods that can predict achievement of key dietary recommendations: data mining of the UK National Diet and Nutrition Survey, 2008-12
}

\author{
Philippe J Giabbanelli† and Jean Adams* \\ UKCRC Centre for Diet and Activity Research (CEDAR), MRC Epidemiology Unit, University of Cambridge School of \\ Clinical Medicine, Institute of Metabolic Science, Cambridge CB2 OQQ, UK
}

Submitted 22 October 2015: Final revision received 21 December 2015: Accepted 19 January 2016: First published online 16 February 2016

\begin{abstract}
Objective: Many dietary assessment methods attempt to estimate total food and nutrient intake. If the intention is simply to determine whether participants achieve dietary recommendations, this leads to much redundant data. We used data mining techniques to explore the number of foods that intake information was required on to accurately predict achievement, or not, of key dietary recommendations.

Design: We built decision trees for achievement of recommendations for fruit and vegetables, sodium, fat, saturated fat and free sugars using data from a national dietary surveillance data set. Decision trees describe complex relationships between potential predictor variables (age, sex and all foods listed in the database) and outcome variables (achievement of each of the recommendations).

Setting: UK National Diet and Nutrition Survey (NDNS, 2008-12).

Subjects: The analysis included 4156 individuals.

Results: Information on consumption of 113 out of 3911 (3\%) foods, plus age and sex was required to accurately categorize individuals according to all five recommendations. The best trade-off between decision tree accuracy and number of foods included occurred at between eleven (for fruit and vegetables) and thirty-two (for fat, plus age) foods, achieving an accuracy of $72 \%$ (for fat) to $83 \%$ (for fruit and vegetables), with similar values for sensitivity and specificity.

Conclusions: Using information on intake of 113 foods, it is possible to predict with $72-83 \%$ accuracy whether individuals achieve key dietary recommendations. Substantial further research is required to make use of these findings for dietary assessment.
\end{abstract}

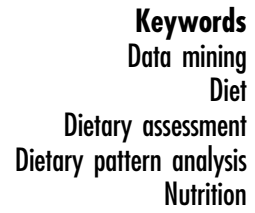

The intention of many dietary assessment methods is to capture information on all foods consumed, or at least those believed to make the largest contribution to total intake $^{(1)}$, in order to estimate total nutrient intake. For some purposes, this detailed estimation of total nutrient intake may lead to collection of much redundant data. This is particularly the case when assessing adherence with policy targets and messages such as 'five-a-day' portions of fruit and vegetables.

The collection of substantial redundant information places unnecessary burden on research participants and unnecessarily uses scarce research resources. To take a first step to overcoming this problem, we applied data mining techniques to explore how many, and which, foods intake information was required on to

$\dagger$ Current affiliation: Department of Computer Science, Northern Illinois University, DeKalb, IL, USA.

*Corresponding author: Email: jma79@medschl.cam.ac.uk

(C) The Authors 2016. This is an Open Access article, distributed under the terms of the Creative Commons Attribution licence (http:// creativecommons.org/licenses/by/4.0/), which permits unrestricted re-use, distribution, and reproduction in any medium, provided the original work is properly cited. accurately predict achievement, or not, of key dietary recommendations.

\section{Data mining, an overview}

Unlike traditional statistical approaches such as multiple regression, data mining allows multiple non-linear relationships and interaction effects to be efficiently captured $^{(2,3)}$. Several data mining tools exist. In the present study we used 'classifiers'. A classifier is a function that labels individuals on an outcome (e.g. achieving a dietary recommendation or not) based on a group of predictor variables (e.g. how much of each individual food was consumed). The analysis package is first provided with a 'training set' of individual-level data in which both the outcome and the predictor variables are known, and uses this to learn how the predictor variables are related to the outcome. This produces the classifier function, which can then be used to infer the outcome in a new case based on 
just the predictor variables. Finally, the accuracy of the classifier is evaluated on a new 'testing set' of data.

There are numerous ways to build classifiers. We used 'decision trees' ${ }^{, 2,4,5)}$. Decision trees provide a graphical illustration of a classifier composed of a number of predictor variables. A decision tree involves repeated 'cuts' of the data according to the level of included predictor variables to identify groups of individuals who are similar in terms of the outcome variable of interest. This produces a decision tree where the path from the root to the outcome corresponds to successive 'cuts', or divisions, of the population.

Figure 1 provides a simplified, hypothetical example of a decision tree where the intention is to identify whether or not individuals achieve the recommended intake of fruit and vegetables (the outcome) using information on the consumption of carrots and white bread (the two predictor variables). Figure 1(a) shows the decision tree based on the 'cuts' represented in Fig. 1(b), the latter being a simple graphical plot of consumption of both carrots and white bread with all individuals labelled according to whether or not they achieve the recommended intake of fruit and vegetables. In terms of meeting fruit and vegetable recommendations there appear to be five 'clusters' of participants in Fig. 1(b). A series of 'cuts' can isolate these clusters. The first cut (labelled 'A' in Fig. 1(a) and 1(b)) divides the population according to consumption of carrots. The next two cuts (labelled 'B' and ' $\mathrm{C}$ ') then divide the resulting two groups according to consumption of white bread. Finally, a fourth cut (labelled 'D') divides those with a medium carrot and medium white bread intake according to a more fine-grained assessment of carrot intake.

To build decision trees with different numbers of predictor variables, the minimum number of individual cases that can be further divided by a subsequent 'cut' is varied. If a small group of individuals can be further subdivided, a sizeable tree including many predictor variables can result. However, if limits are placed on the minimum size of group that can be further subdivided, a smaller decision tree, including fewer predictor variables, results. In the current study we made use of this feature to explore the effect of including more or fewer predictor variables on the accuracy of decision trees.

A small number of studies have applied data mining techniques to nutritional data. These have focused primarily on dietary pattern analysis, exploring which dietary components are predictive of a range of health outcomes $^{(6-9)}$. However, we are not aware of any other uses of data mining to identify which foods are predictive of achievement, or not, of key dietary recommendations.

\begin{abstract}
Aim
Our aim was to use data mining techniques to determine the number of foods that intake information was required on to accurately predict achievement, or not, of dietary recommendations for intake of fruit and vegetables, free sugars, sodium, fat and saturated fat.
\end{abstract}

\section{Methods}

We built decision trees for achievement of key dietary recommendations using data from the first four years of the rolling programme of the UK's national dietary surveillance data set: the National Diet and Nutrition Survey (NDNS).

\section{Data source}

The NDNS is an annual cross-sectional survey assessing the diet, nutrient intake and nutritional status of the general population aged 18 months and upwards living in private households in the $\mathrm{UK}^{(10)}$. Since 2008 an annual 'rolling programme' has been in place, allowing data to be combined over years. We used data from years 1-4 of this programme, collected in 2008-12.

The NDNS aims to collect data from a sample of 1000 respondents per year: at least 500 adults (aged 19 years and (a)

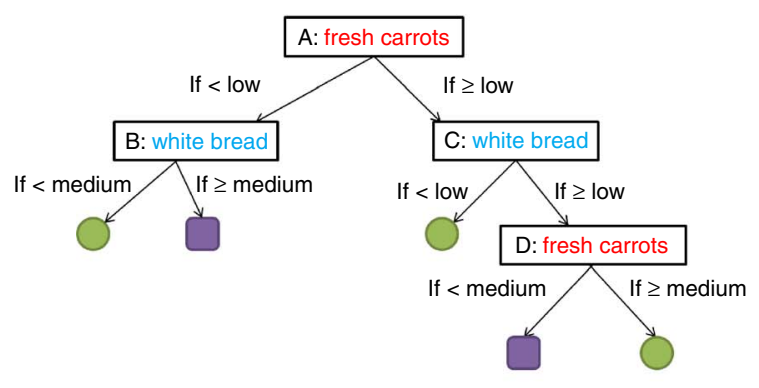

(b)

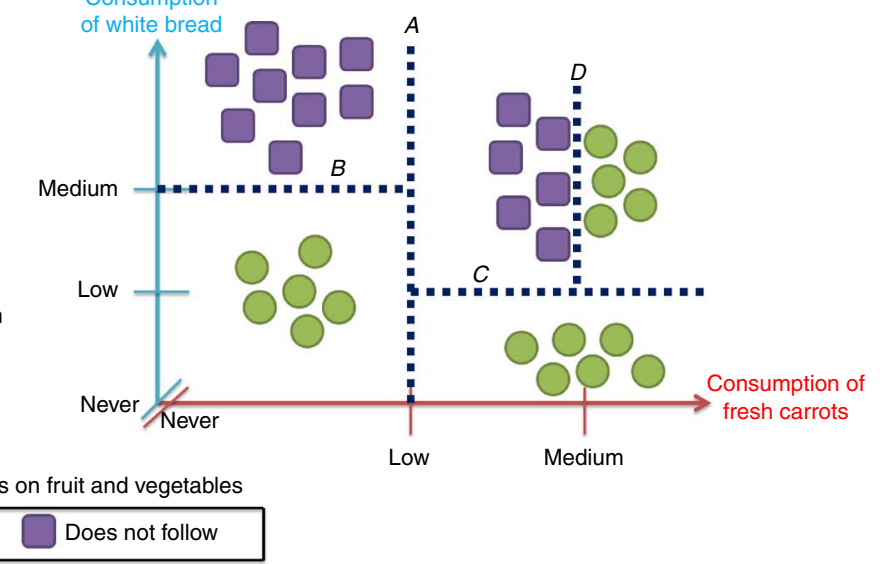

Recommendations on fruit and vegetables

Fig. 1 (colour online) Schematic illustration of a decision tree (a) and how this is formed through repeated 'cuts' of the data (b) 
older) and at least 500 children (aged 1.5 to 18 years). Households across the UK are selected to take part in the NDNS using a multistage probability design. In each wave, a random sample of primary sampling units is selected for inclusion. These are small geographical areas that allow more efficient data collection by enabling it to be geographically focused. Within these primary sampling units, private addresses are randomly selected for inclusion. If, on visiting, it is found that more than one household lives at a particular address, one is randomly selected for inclusion. Within participating households, up to one adult and one child are randomly selected to take part as 'respondents'. Data collection includes completion of a $4 \mathrm{~d}$ estimated food diary, where participants estimate the weight of foods consumed using food labels and household measures ${ }^{(11)}$.

NDNS data were obtained from the UK Data Archive, an online resource that makes research data available to the UK research community.

\section{Inclusion and exclusion criteria}

NDNS participants were included in the analysis if they completed 3 or $4 \mathrm{~d}$ of the estimated food diary. As recommendations for fruit and vegetable intake apply only to those aged 11 years or older, children aged less than 11 years were excluded from this component of the analysis.

\section{Outcomes of interest: achievement of dietary recommendations}

Information on which foods were consumed, and how much participants estimated was consumed, was combined with nutritional information to determine mean daily intake of fruit and vegetables ( $80 \mathrm{~g}$ portions) and sodium (milligrams), as well as mean daily percentage of energy derived from fat, saturated fat and free sugars, for each individual. This information was then used to determine whether or not each individual met international, or UK, recommendations for these variables.

We used UK recommendations for fruit and vegetable and sodium intakes, as these have been graded according to age. It is recommended that individuals aged 11 years and older consume at least five $80 \mathrm{~g}$ portions of fruit and vegetables daily. This includes a maximum of one portion of juice, with additional juice portions not counted. For sodium, current UK recommendations are that those aged 11 years and older consume no more than $2400 \mathrm{mg} / \mathrm{d}$; children aged $7-10$ years, no more than $2000 \mathrm{mg} / \mathrm{d}$; children aged 4-6 years, no more than $1200 \mathrm{mg} / \mathrm{d}$; and children aged $1-3$ years, no more than $800 \mathrm{mg} / \mathrm{d}^{(12)}$.

The WHO recommends population food and nutrient intake goals for the avoidance of diet-related diseases. These state that no more than $30 \%$ of energy should be derived from fat, no more than $10 \%$ from saturated fat and no more than $10 \%$ from free sugars ${ }^{(13)}$.
Predictor variables of interest: foods consumed

In total, 3911 different foods (including drinks) have been recorded in NDNS food diaries. We used total estimated weight (in grams) of each individual food eaten by each individual as potential predictor variables. Age and sex were also included as potential predictor variables. The use of including markers of socio-economic position (education, income and social class) as potential predictor variables was explored, but these were found to add no additional increase in accuracy over and above age, sex and individual foods. Decision trees reported here do not include any socio-economic predictor variables.

\section{Data analysis}

Our analysis scripts and detailed decision trees are available at https://osf.io/znv82. In all cases except sodium, the proportion of individuals achieving the recommendations was substantially less than $50 \%$; for sodium substantially more than $50 \%$ of individuals achieved the recommendations (Table 2). As detailed in the online supplementary material, this imbalance in outcome variables can lead to low-quality classifiers. To correct this, we pre-processed the data using the Synthetic Minority Over-sampling TEchnique (SMOTE) ${ }^{(14)}$, which creates new cases for the group that accounted for less than $50 \%$ of participants by interpolating between existing cases that lie together. WEKA software ${ }^{(15)}$ was then used to build decision trees using the $\mathrm{J} 48$ algorithm and error pruning.

For each outcome of interest we built a series of decision trees with different numbers of predictor variables by varying the minimum number of individual cases that could be further divided. For each of the decision trees built, we calculated the number of predictor variables used and overall accuracy in correctly classifying individuals. We used the standard tenfold cross-validation procedure $^{(16)}$ in which the entire eligible NDNS data set was split into ten approximately equally sized parts. Nine parts were used in turn as training sets and the remaining tenth part was used as the testing set. The ability of decision trees to correctly identify those who achieved the recommendations (sensitivity) and those who did not (specificity) was also calculated. Adaptive sampling was used to identify the maximum overall accuracy that could be achieved, as well as the optimum trade-off between minimizing the number of predictor variables and maximizing the overall accuracy.

\section{Results}

Overall, $91 \%$ of households eligible for inclusion agreed to take part in the first four waves of NDNS. Within these, $56 \%$ (2083 adults and 2073 children; 4156 participants in total) of individuals selected to take part completed 3 or $4 \mathrm{~d}$ of the estimated food diary and were included in the 
Table 1 Comparison of the analytical sample with the UK population

\begin{tabular}{|c|c|c|c|c|c|c|c|c|}
\hline \multirow[b]{3}{*}{ Variable } & \multicolumn{4}{|c|}{ Adults aged 19 years or older } & \multicolumn{4}{|c|}{ Children aged $<19$ years } \\
\hline & \multicolumn{2}{|c|}{ Analytical sample (n 2083) } & \multicolumn{2}{|c|}{ UK population } & \multicolumn{2}{|c|}{ Analytical sample (n 2073) } & \multicolumn{2}{|c|}{ UK population } \\
\hline & $n$ & $\%$ & $n$ & $\%$ & $n$ & $\%$ & $n$ & $\%$ \\
\hline Female & 1182 & $56 \cdot 8$ & 25198773 & 51.5 & 1007 & $48 \cdot 6$ & 6955262 & $48 \cdot 8$ \\
\hline \multicolumn{9}{|l|}{ Age (adults) } \\
\hline $19-29$ years & 296 & 14.2 & 9447071 & $19 \cdot 3$ & \multicolumn{2}{|c|}{ - } & \multicolumn{2}{|c|}{ - } \\
\hline $30-39$ years & 390 & $18 \cdot 7$ & 8319926 & $17 \cdot 0$ & \multicolumn{2}{|c|}{-} & \multicolumn{2}{|c|}{-} \\
\hline 40-49 years & 425 & $20 \cdot 4$ & 9268735 & 18.9 & \multicolumn{2}{|c|}{-} & \multicolumn{2}{|c|}{-} \\
\hline $50-59$ years & 363 & 17.4 & 7708532 & $15 \cdot 8$ & \multicolumn{2}{|c|}{-} & \multicolumn{2}{|c|}{ - } \\
\hline 60-64 years & 181 & 8.7 & 3807975 & $7 \cdot 8$ & \multirow{2}{*}{\multicolumn{2}{|c|}{-}} & \multicolumn{2}{|c|}{ - } \\
\hline$\geq 65$ years & 428 & $20 \cdot 6$ & 10377127 & 21.2 & & & & \\
\hline \multicolumn{9}{|l|}{ Age (children) } \\
\hline $0-4$ years & \multicolumn{2}{|c|}{-} & \multicolumn{2}{|c|}{-} & 499 & $24 \cdot 1$ & 3913953 & 27.5 \\
\hline 5-9 years & \multirow{2}{*}{\multicolumn{2}{|c|}{$\begin{array}{l}- \\
-\end{array}$}} & \multirow{2}{*}{\multicolumn{2}{|c|}{-}} & 583 & $26 \cdot 4$ & 3516615 & 24.7 \\
\hline $10-14$ years & & & & & 547 & $26 \cdot 4$ & 3669326 & $25 \cdot 7$ \\
\hline $15-18$ years & \multicolumn{2}{|c|}{-} & \multicolumn{2}{|c|}{-} & 444 & 21.4 & 3152919 & $22 \cdot 1$ \\
\hline
\end{tabular}

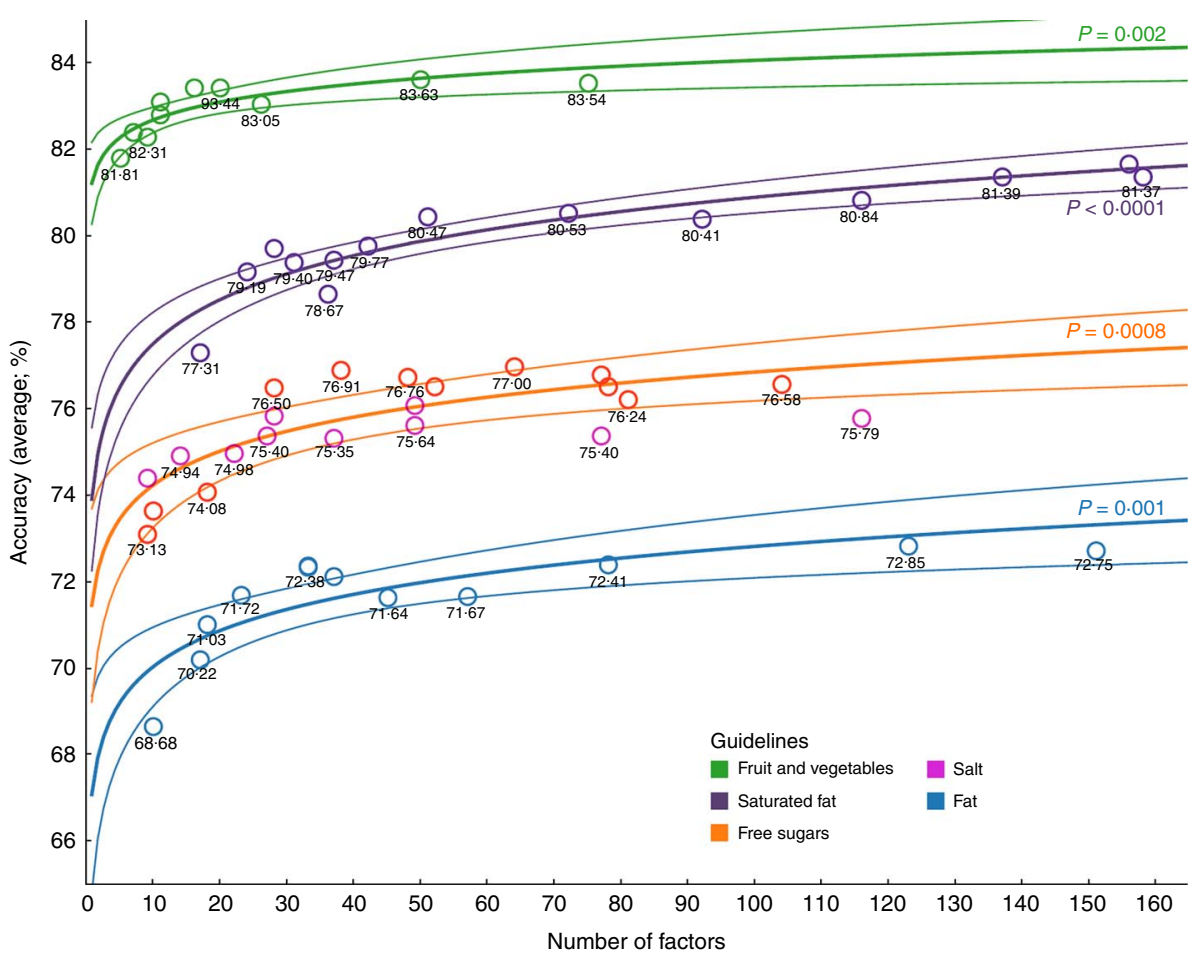

Fig. 2 (colour online) Overall accuracy (with 95\% confidence margins) of decision trees $v$. the number of predictor variables included, using data mining techniques on the nutritional intake of 4156 individuals (2967 individuals for fruit and vegetables) from the UK National Diet and Nutrition Survey (2008-12)

analysis for sodium, free sugars, fat and saturated fat. Of these 4156 participants, 2967 (71.4\%) were aged 11 years or older and included in the analysis for fruit and vegetables. There were no missing data on sex or age.

The distributions of age and sex in the analytical sample compared with the UK population as a whole are shown in Table 1. As the NDNS sample contains relatively equal numbers of children aged 18 years or younger and adults, distributions are provided separately for adults and children in Table 1 . The main differences in the age and sex distributions between the analytical sample and the UK population were that the analytical sample had a higher proportion of adult women and a lower proportion of young adults (aged 19-29 years) than the UK population.

Figure 2 shows the overall accuracy of decision trees for each of the five outcomes plotted against the number of predictor variables in decision trees. Overall accuracy ranged from $69 \%$ (fat; ten predictor variables) to $84 \%$ (fruit and vegetables; fifty predictor variables) depending on the outcome of interest and number of predictor 
variables included. For all guidelines but sodium, the relationship between the number of predictor variables and the accuracy was best described using a logarithmic trend model $(P<0 \cdot 01$ in all cases). Thus, increasing the number of predictor variables from about ten to thirty improved the accuracy by a maximum of about five percentage points, but beyond this adding even a large number of additional predictor variables yielded only a very small additional improvement. We were unable to fit any function to the relationship between accuracy and number of predictor variables for sodium.

Table 2 provides information on the decision tree for each outcome that represented the best trade-off between accuracy and number of predictor variables. Information on the most accurate possible tree for each outcome is also shown in Table 2. Between eleven (for fruit and vegetables) and thirty-three (for fat) predictor variables provided the best trade-off to identify whether individuals achieved each of the recommendations, achieving overall accuracy of $72 \%$ (for fat) to $83 \%$ (for fruit and vegetables). Adding further predictor variables beyond this improved accuracy by a maximum of $2 \%$ (for saturated fat) and less than $1 \%$ (for all other outcomes). Sensitivity and specificity were similar to overall accuracy for fruit and vegetables and free sugars (and for saturated fat when the maximum number of predictor variables was included). However, specificity was higher than sensitivity for fat (and saturated fat), but the reverse was seen for sodium. Predictor variables in decision trees with the best trade-off between accuracy and number of predictor variables accounted for between $13 \%$ (for fat) and 31\% (for free sugars) of total intake of relevant outcome variables.
Predictor variables used in decision trees with the best trade-off between accuracy and number of predictor variables are shown in Table 3. In total, 113 foods (3\% out of a total 3911 recorded as consumed), age and sex were included in the decision trees for all five outcomes. Overall, there was little overlap in predictor variables across outcomes. Age and two foods were included as predictor variables in the decision trees for three outcomes. A further six foods were included as predictor variables in the decision trees for two outcomes. The remaining 104 foods were included as predictor variables in only one decision tree.

\section{Discussion}

\section{Summary of results}

The present study represents the first work we are aware of using data mining techniques to explore the number of foods that information is required on to predict achievement of dietary recommendations. In total, information on consumption of 113 of 3911 foods (3\%), plus age and sex was required to accurately categorize individuals according to all five dietary recommendations (fruit and vegetables, free sugars, sodium, fat and saturated fat). The best trade-off between decision tree accuracy and number of foods included was achieved at between eleven (for fruit and vegetables) and thirty-two (for fat, plus age) foods. These decision trees had an overall accuracy of $72 \%$ (for fat) to $83 \%$ (for fruit and vegetables), with similar values for sensitivity and specificity. Few individual foods were present

Table 2 Prevalence of achieving and not achieving dietary recommendations and accuracy of decision trees to predict this, using data mining techniques on the nutritional intake of 4156 individuals (2967 individuals for fruit and vegetables) from the UK National Diet and Nutrition Survey (2008-12)

\begin{tabular}{|c|c|c|c|c|c|}
\hline & Fruit \& vegetables & Free sugars & Sodium & Fat & Saturated fat \\
\hline No. achieving recommendation without oversampling & 656 & 1472 & 2524 & 1045 & 795 \\
\hline$\%$ & $22 \cdot 1$ & 35.4 & $60 \cdot 7$ & $25 \cdot 1$ & $19 \cdot 1$ \\
\hline SMOTE oversampling \%* & $252 \%$ (yes) & $85 \%$ (yes) & $54 \%(\mathrm{no})$ & $197 \%$ (yes) & $322 \%$ (yes) \\
\hline No. achieving recommendation after oversampling & $2309^{*}$ & 2679 & 2524 & 3103 & 3354 \\
\hline No. not achieving recommendation after oversampling & $2311^{*}$ & 2684 & 2513 & 3111 & 3361 \\
\hline \multicolumn{6}{|c|}{ Decision tree with the best trade-off between accuracy and number of predictor variables } \\
\hline Overall accuracy (\%) & 83.1 & 76.5 & 75.9 & $72 \cdot 4$ & $79 \cdot 7$ \\
\hline Sensitivity (\%) & $82 \cdot 5$ & $76 \cdot 1$ & 81.9 & $66 \cdot 3$ & $75 \cdot 8$ \\
\hline Specificity (\%) & $83 \cdot 8$ & $76 \cdot 9$ & $69 \cdot 8$ & 78.4 & $83 \cdot 6$ \\
\hline No. of predictor variables & 11 & 28 & 28 & 33 & 28 \\
\hline $\begin{array}{l}\% \text { of all relevant food/nutrient }(\mathrm{g}) \text { accounted for by predictor } \\
\text { variables }\end{array}$ & $21 \cdot 0 \dagger$ & $31 \cdot 2$ & $13 \cdot 4$ & $13 \cdot 0$ & $27 \cdot 4$ \\
\hline \multicolumn{6}{|l|}{ Most accurate decision tree } \\
\hline Overall accuracy $(\%)$ & 83.6 & $77 \cdot 0$ & $76 \cdot 1$ & $72 \cdot 9$ & $81 \cdot 7$ \\
\hline Sensitivity (\%) & 83.9 & $75 \cdot 7$ & $80 \cdot 7$ & $69 \cdot 3$ & 81.4 \\
\hline Specificity (\%) & $83 \cdot 3$ & $78 \cdot 3$ & 71.5 & $76 \cdot 4$ & 81.9 \\
\hline No. of predictor variables & 50 & 64 & 49 & 123 & 156 \\
\hline $\begin{array}{l}\% \text { of all relevant food } / \text { nutrient }(\mathrm{g}) \text { accounted for by predictor } \\
\text { variables }\end{array}$ & $30 \cdot 8 \dagger$ & $38 \cdot 6$ & $25 \cdot 4$ & 29.5 & $42 \cdot 7$ \\
\hline
\end{tabular}

SMOTE, Synthetic Minority Over-sampling TEchnique.

*After oversampling using the SMOTE method (see online supplementary material).

†Percentage of all fruit and vegetables (g) recorded, not just those contributing to 5 -a-day portions (specifically, fruit juice can contribute a maximum of only one 5-a-day portion). 
Table 3 Predictor variables (individual foods, age and sex) included in decision trees for predicting achievement of five dietary recommendations, using data mining techniques on the nutritional intake of 4156 individuals (2967 individuals for fruit and vegetables) from the UK National Diet and Nutrition Survey (2008-12)

\begin{tabular}{|c|c|c|c|c|c|}
\hline \multicolumn{5}{|c|}{ Dietary recommendation outcome } & \multirow[b]{2}{*}{ Food name } \\
\hline Fat & Free sugars & Fruit \& vegetables & Sodium & Saturated fat & \\
\hline Yes & & & Yes & Yes & Age \\
\hline \multirow{5}{*}{ Yes } & & & & & Alcoholic soft drinks, spirit based \\
\hline & & Yes & & & Almonds, kernel only: ground almonds \\
\hline & Yes & & & & Apple juice, unsweetened, cartons, pasteurized \\
\hline & Yes & & & & Apple juice, unsweetened, UHT \\
\hline & & Yes & & & Apples, eating, raw, flesh \& skin only \\
\hline \multirow{4}{*}{ Yes } & & & & & Avocado pear, flesh only \\
\hline & & & Yes & & Bacon rashers, back, grilled, lean and fat \\
\hline & & & Yes & & Bacon rashers, back, not smoked, grilled, extra trim \\
\hline & & & Yes & & Baked beans in tomato sauce with pork sausages \\
\hline \multirow[t]{2}{*}{ Yes } & & Yes & & & Bananas, raw, flesh only \\
\hline & & & & Yes & Beefburger and onion, grilled \\
\hline \multirow[t]{5}{*}{ Yes } & & & & & Black pudding, fried \\
\hline & Yes & & & & Blackcurrant juice drink, ready to drink, not low calorie \\
\hline & Yes & & & & Boiled sweets, barley sugar, butterscotch, glacier mints, hard candy \\
\hline & & & Yes & & Bread, white, crusty \\
\hline & & & Yes & Yes & Bread, white, toasted \\
\hline \multirow[t]{4}{*}{ Yes } & & & & & Bread, $50 \%$ white and $50 \%$ wholemeal flours \\
\hline & & & Yes & & Bread, white sliced, not fortified \\
\hline & & & Yes & & Brown sauce, bottled \\
\hline & & & Yes & & Brussels sprouts, fresh, boiled \\
\hline Yes & & & & & Butter beans, dried, boiled \\
\hline \multirow[t]{3}{*}{ Yes } & & & & Yes & Butter, salted \\
\hline & & & & Yes & Butter, unsalted \\
\hline & Yes & & & & Carbonated beverages, no juice, not low calorie, canned \\
\hline \multirow[t]{2}{*}{ Yes } & Yes & & & Yes & Carbonated beverages, no juice, not low calorie, not canned \\
\hline & & Yes & & & Celery, fresh, raw \\
\hline Yes & & & & & Chapatti, brown, no fat \\
\hline \multirow{3}{*}{ Yes } & & & & Yes & Cheese, cheddar, any other or for recipes \\
\hline & & & & Yes & Cheese, cheddar, English \\
\hline & & & Yes & & Cheese, soft full fat, Philadelphia type \\
\hline \multirow[t]{7}{*}{ Yes } & & & & & Chicken fried in olive oil \\
\hline & & & & Yes & Children's fromage frais fruit with added vitamin D \\
\hline & & & & Yes & Chocolate brownie, no nuts, purchased \\
\hline & & & & Yes & Chocolate-covered caramels, Cadbury Caramel \\
\hline & Yes & & & & Chocolate Swiss roll with butter cream, purchased \\
\hline & Yes & & & & Cola cherry cola, canned, not low calorie \\
\hline & Yes & & & & Cola, not canned, not low calorie, not caffeine free \\
\hline Yes & & & & & Coleslaw, purchased, not low calorie \\
\hline \multirow[t]{12}{*}{ Yes } & & & & & Cookies and biscuits with chocolate \\
\hline & & & & Yes & Cornetto type ice cream, chocolate or nut based \\
\hline & Yes & & & & Cranberry fruit juice drink, e.g. Ocean Spray \\
\hline & & & & Yes & Cream, double \\
\hline & Yes & & & & Cream egg \\
\hline & & & & Yes & Croissants, plain, not filled \\
\hline & Yes & & & & Drinking chocolate, instant, dry weight \\
\hline & & & Yes & & Fat spread $(62-72 \%$ fat), not polyunsaturated \\
\hline & Yes & & & & Fruit gums, wine gums \\
\hline & Yes & & & & Fruit juice drink, carbonated, not low calorie, not canned \\
\hline & Yes & & & & Fruit juice drink with $5 \%$ fruit juice, ready to drink \\
\hline & & & & Yes & Fully coated chocolate biscuits with biscuit filling \\
\hline Yes & & & & & Garlic bread, lower fat \\
\hline & & & Yes & & Ham, unspecified, not smoked, not canned \\
\hline & & & Yes & & Hamburger, Big Mac, McDonalds \\
\hline & Yes & & & & High juice, ready to drink, not blackcurrant or low calorie \\
\hline & Yes & & & & Ice lollies \\
\hline & Yes & & & & Jaffa Cakes \\
\hline & & & & Yes & Kit Kat \\
\hline Yes & & & & & Lager, not canned, e.g. Heineken \\
\hline Yes & & & & & Lager, not canned, e.g. Skol \\
\hline Yes & & & & & Lamb scrag and neck, stewed, lean only \\
\hline & Yes & & & & Lemonade, not low calorie, not canned \\
\hline & & & & Yes & Light spreadable butter ( $60 \%$ fat) \\
\hline & Yes & & & & Lucozade sport isotonic drink, not carbonated \\
\hline Yes & & & & Yes & Mayonnaise (retail) \\
\hline & & & Yes & Yes & Milk chocolate bar \\
\hline
\end{tabular}


Table 3 Continued

\begin{tabular}{|c|c|c|c|c|c|}
\hline \multicolumn{5}{|c|}{ Dietary recommendation outcome } & \multirow[b]{2}{*}{ Food name } \\
\hline Fat & Free sugars & Fruit \& vegetables & Sodium & Saturated fat & \\
\hline & Yes & & & & Milk shake, thick style, takeaway \\
\hline \multirow[t]{2}{*}{ Yes } & & & & Yes & $\begin{array}{l}\text { Milk, skimmed, after boiling } \\
\text { Milk, whole pasteurized, winter }\end{array}$ \\
\hline & & & & Yes & Milk, whole pasteurized, summer \\
\hline \multirow[t]{6}{*}{ Yes } & & & & & Mushrooms fried in olive oil \\
\hline & & & Yes & & Naan bread, plain \\
\hline & & Yes & & & Oatcakes \\
\hline & Yes & & & & Olive oil \\
\hline & & Yes & & & Onions, boiled \\
\hline & Yes & & & & Orange juice, unsweetened, UHT \\
\hline \multirow[t]{2}{*}{ Yes } & & & & & Oven ready chips \\
\hline & & & Yes & & Papadums/poppadoms, fried in vegetable ghee \\
\hline \multirow[t]{2}{*}{ Yes } & & & & & Pasta noodles, boiled \\
\hline & & & Yes & & Pasta noodles, egg, boiled \\
\hline \multirow[t]{3}{*}{ Yes } & & & & & Pasta spaghetti, boiled, white \\
\hline & & & Yes & & Peanut butter, crunchy, not wholenut \\
\hline & & Yes & & & Pears, eating, raw, flesh \& skin only, no core \\
\hline \multirow[t]{3}{*}{ Yes } & & & & & Pepperami \\
\hline & & & & Yes & Petit Filous fromage frais \\
\hline & & & Yes & & Potato cakes (scones), purchased \\
\hline \multirow[t]{8}{*}{ Yes } & & & & & Potatoes, new, boiled, skins eaten \\
\hline & & & Yes & & Potatoes, old, baked, flesh \& skin \\
\hline & & & & Yes & Potatoes, old, mashed \& butter \\
\hline & & & Yes & & Prawns, boiled, flesh only \\
\hline & & & Yes & & Reduced fat spread $(41-62 \%)$, not polyunsaturated \\
\hline & & & Yes & & Ribena, original blackcurrant drink, concentrate \\
\hline & Yes & & & & Robinsons Fruit Shoot \\
\hline & & & Yes & & Rolls, white, crusty \\
\hline \multirow{3}{*}{$\begin{array}{l}\text { Yes } \\
\text { Yes }\end{array}$} & & Yes & & Yes & Sausage roll, flaky pastry, purchased \\
\hline & & & & & Sausages, pork, grilled \\
\hline & & & Yes & & Sausages, premium pork, grilled \\
\hline \multirow{2}{*}{$\begin{array}{l}\text { Yes } \\
\text { Yes }\end{array}$} & & & & & Scrambled eggs with skimmed milk and no fat \\
\hline & & & & & Semi-sweet biscuit \\
\hline & & & Yes & & Sex \\
\hline & Yes & & & & Soya alternative to milk, sweetened plain \\
\hline & & Yes & & & Spinach, fresh, raw \\
\hline & & & & Yes & Spreadable butter ( $75-80 \%$ fat) \\
\hline & Yes & & & & Sugar, white \\
\hline & & & & Yes & Super Noodles, Batchelors, as served \\
\hline & & & & Yes & Swiss roll, individual, chocolate coated, purchased \\
\hline & & Yes & & & Tomatoes, raw \\
\hline & & & Yes & & Turkey slices, unsmoked, pre-pack or deli \\
\hline & Yes & & & & Water for concentrated soft drinks, not diet \\
\hline Yes & & & & & White chocolate buttons, mice \\
\hline Yes & & & & & Whole milk, after boiling \\
\hline Yes & & & & & Wine white, dry, not canned \\
\hline & Yes & & & Yes & Yoghurt twin pot with cereal/crumble \\
\hline & & Yes & & & Yoghurt, Greek style, cows, natural, whole milk \\
\hline & & & Yes & & Yorkshire pudding, frozen \\
\hline
\end{tabular}

UHT, ultra-heat treated.

in the decision tree for more than one dietary recommendation, although age was present in three.

\section{Strengths and limitations of methods}

We used data from a population-based sample, meaning that our findings are likely to be generalizable across the UK and to other countries with similar dietary profiles. However, diets vary internationally ${ }^{(17)}$ and our results may not be more widely generalizable. The analytical sample had a slightly higher proportion of adult women and a lower proportion of younger adults (aged 19-29 years) than the UK population as a whole.

The data used were collected using 'estimated' food diaries where portion sizes were estimated but not weighed. These are considered to be one of the more accurate methods of measuring dietary intake ${ }^{(18)}$, meaning that both the predictor and outcome variables are likely to be valid. However, even estimated food diaries have their limitations, particularly in terms of participant burden and under-reporting of energy intake ${ }^{(19,20)}$. Doubly labelled water has been used to estimate total energy expenditure 
in a sub-sample of NDNS participants and compared with reported energy intake from food diaries. This reveals that reported energy intake is $12-34 \%$ lower than estimated total energy expenditure, depending on the age of participants ${ }^{(11)}$. This mismatch may be due to intentional or unintentional misreporting; participants changing their food intake in response to recording it; or a variety of other reasons. However, misreporting is unlikely to affect all foods and nutrients equally. For example, participants may be more likely to misreport confectionery than vegetable intake. For this reason, misreporting is not adjusted for in NDNS and we have not adjusted for misreporting here.

Data mining using decision trees is computationally and statistically efficient. For example, inclusion of all 3911 foods consumed by NDNS participants in regression models with achievement of dietary recommendations as outcomes would be computationally, and statistically, demanding and unlikely to produce satisfactory results. Decision trees also produce transparent, and intuitively understandable, outputs (ours are provided at https://osf.io/znv82) ${ }^{(21)}$.

Many of foods included in the analysis had very skewed distributions. Indeed, the vast majority of foods in the database (3618) were eaten by less than 150 people. Decision trees seek to maximize information gain at each step, rather than working with the distribution as a whole as in traditional regression analysis. If an item is very discriminatory and helps differentiate between those who do and do not meet a particular guideline then it will be included, even if it is consumed by only a small number of people. Conversely, if an item is eaten by almost everyone but is not discriminatory, then it would be unlikely to be included. There was no overall trend between the proportion of participants who ate a food and the chance that that food was included in a decision tree (data not shown).

We used adaptive sampling to identify decision trees that achieved the best trade-off between accuracy and number of predictor variables included. Thus, instead of systematically calculating the accuracy of all decision trees including all possible number of predictor variables, we focused on identifying the relationship between accuracy and number of predictor variables (logarithmic in most cases) and where the optimum trade-off between accuracy and number of predictor variables occurred (i.e. where the logarithmic curve flattened out). This means we cannot be absolutely sure that we have identified the decision trees with the best trade-off between accuracy and number of predictor variables in all cases. However, given the very small additional improvements in accuracy achieved by the most accurate $v$. best trade-off decision trees, we are certainly likely to have identified the near-best trade-off decision trees.

We used estimated dietary records as our 'gold standard' tool for determining whether or not individuals achieved recommendations. Further work will be required to compare the accuracy of our decision trees with other methods of estimating who achieves dietary recommendations, such as FFQ.

\section{Interpretation and implications of findings and areas for future work}

Our findings indicate that information on only a small number of foods is required to determine whether individuals achieve five important dietary recommendations. If such binary outcomes are the key outcome of interest, then more detailed dietary assessment methods may inappropriately use scarce research resources and be unnecessarily burdensome to participants.

While our results suggest that information on only a limited number of foods needs to be captured when assessing whether guidelines are met, substantial further research will be needed before these findings could be applied in the form of a new dietary assessment instrument. First, it would be helpful to replicate our analyses in a different, but comparable, sample. We have not done this as we are not aware of a comparable UK population-representative sample in whom diet diaries have been collected. Our decision trees used information on exact intake of 113 foods over 3-4d. Assessing exact intake of a small number of foods may be no less burdensome for participants than assessing estimated intake of all foods using a food diary. Future work could compare the accuracy of decision trees based on exact intake of 113 foods, approximate intake of these foods (e.g. using the ordinal categories often used in FFQ), and exact and approximate intakes of foods at the food group, rather than individual food, level. Acceptability to research participants and resource implications of collecting the data required in all cases should also be compared.

Our analysis focused on which foods can be used to predict whether or not individuals achieve dietary recommendations. But it is not necessarily the case that it is the foods included in the decision tress which cause people to achieve the recommendations or not. A maximum of only $31 \%$ of the total intake of relevant nutrients or foods was accounted for by predictor variables in decision trees with the best trade-off between accuracy and number of predictor variables. Thus, decision trees did not particularly include foods that account for the majority of intake of nutrients and foods of interest - as might be expected in an FFQ. The complex relationships between individual foods included in our decision trees and the dietary recommendations they are associated with may offer further useful insights and could be studied further.

\section{Conclusion}

We used data mining techniques to explore the number of foods that consumption information was required on to accurately predict achievement, or not, of five key dietary 
recommendations. Information on consumption of eleven to thirty-two foods (plus age and sex) was sufficient to identify with $72-83 \%$ accuracy whether individuals achieved individual dietary recommendations. In total, information on 113 foods was required to predict achievement of all five recommendations studied. This method could be used to develop a new dietary assessment questionnaire.

\section{Acknowledgements}

Acknowledgements: Thanks are extended to Simon Wheeler at the MRC Epidemiology Unit, Cambridge University for help with interpreting NDNS data. Financial support: The work was undertaken by the Centre for Diet and Activity Research (CEDAR), a UKCRC Public Health Research Centre of Excellence. Both authors gratefully acknowledge funding from the British Heart Foundation, Cancer Research UK, the Economic and Social Research Council, the Medical Research Council (MRC), the National Institute for Health Research (NIHR) and the Wellcome Trust, under the auspices of the UK Clinical Research Collaboration (reference MR/K023187/ 1). Conflict of interest: None. Authorship: J.A. conceived the original idea for this work. P.J.G. designed and conducted the data analysis, and produced all figures and tables. Both authors interpreted the results and drafted the manuscript. Ethics of human subject participation: Ethical approval for the NDNS was obtained from the Oxfordshire A Research Ethics Committee and all participants provided informed consent to take part in the survey. Further ethical approval was not required for the current secondary analysis of anonymized data.

\section{Supplementary material}

To view supplementary material for this article, please visit http://dx.doi.org/10.1017/S1368980016000185

\section{References}

1. Willett WC, Sampson L, Stampfer MJ et al. (1985) Reproducibility and validity of a semiquantitative food frequency questionnaire. Am J Epidemiol 122, 51-65.

2. Crutzen R \& Giabbanelli P (2013) Using classifiers to identify binge drinkers based on drinking motives. Subst Use Misuse (Epublication ahead of print version).

3. Dierker L, Rose J, Tan X et al. (2010) Uncovering multiple pathways to substance use: a comparison of methods for identifying population subgroups. J Prim Prev 31, 333-348.

4. McKenzie DP, McFarlane AC, Creamer M et al. (2006) Hazardous or harmful alcohol use in Royal Australian Navy veterans of the 1991 Gulf War: identification of high risk subgroups. Addict Behav 31, 1683-1694.

5. Hillemacher T, Frieling H, Wilhelm J et al. (2012) Indicators for elevated risk factors for alcohol-withdrawal seizures: an analysis using a random forest algorithm. J Neural Transm 119, 1449-1453.

6. Lazarou C, Karaolis M, Matalas A-L et al. (2012) Dietary patterns analysis using data mining method. An application to data from the CYKIDS study. Comput Methods Programs Biomed 108, 706-714.

7. Kastorini C-M, Papadakis G, Milionis HJ et al. (2013) Comparative analysis of a-priori and a-posteriori dietary patterns using state-of-the-art classification algorithms: a case/casecontrol study. Artif Intell Med 59, 175-183.

8. Thangamani D \& Sudha P (2014) Identification of malnutrition with use of supervised datamining techniques - decision trees and artificial neural networks. Int J Eng Comput Sci 3, 8236-8241.

9. Einsele F, Sadeghi L, Ingold R et al. (2015) A study about discovery of critical food consumption patterns linked with lifestyle diseases using data mining methods. In Proceedings of the International Conference on Health Informatics, Lisbon, Portugal, pp. 239-245; available at http://www. scitepress.org/DigitalLibrary/PublicationsDetail.aspx?ID $=\mathrm{h} 6$ vMPttIHy0 $=\& \mathrm{t}=1$

10. Bates B, Lennox A \& Swan G (editors) (2010) National Diet and Nutrition Survey: Headline Results from Year 1 of the Rolling Programme (2008/2009). London: Food Standards Agency and Department of Health.

11. Bates B, Lennox A, Prentice A et al., (editors) (2014) National Diet and Nutrition Survey Results from Years 1, 2, 3 and 4 (combined) of the Rolling Programme (2008/2009-2011/ 2012). London: Public Health England.

12. Scientific Advisory Committee on Nutrition (2003) Salt and Health. London: The Stationery Office.

13. World Health Organization (2003) Diet, Nutrition and the Prevention of Chronic Diseases. Report of a Joint WHO/FAO Expert Consultation. WHO Technical Report Series no. 916. Geneva: WHO.

14. Chawla N, Bowyer K, Hall L et al. (2002) SMOTE: synthetic minority over-sampling technique. J Artif Intell Res 16, 321-357.

15. Bouckaert RR, Frank E, Hall MA et al. (2010) WEKA experiences with a Java open-source project. J Mach Learn Res 11, 2533-2541.

16. Kuncheva L (2004) Fundamentals of Pattern Recognition Combining Pattern Classifiers: Methods and Algorithms. Hoboken, NJ: John Wiley \& Sons.

17. Imamura F, Micha R, Khatibzadeh S et al. (2015) Dietary quality among men and women in 187 countries in 1990 and 2010: a systematic assessment. Lancet Glob Health 3, e132-e142.

18. Bingham S, Gill C, Welch A et al. (1994) Comparison of dietary assessment methods in nutritional epidemiology: weighed records $v .24 \mathrm{~h}$ recalls, food-frequency questionnaires and estimated-diet records. $\mathrm{Br} J \mathrm{Nutr}$ 72, 619-643.

19. Poslusna K, Ruprich J, de Vries JH et al. (2009) Misreporting of energy and micronutrient intake estimated by food records and 24 hour recalls, control and adjustment methods in practice. Br J Nutr 101, Suppl. 2, S73-S85.

20. Burrows TL, Martin RJ \& Collins CE (2010) A systematic review of the validity of dietary assessment methods in children when compared with the method of doubly labeled water. J Am Diet Assoc 110, 1501-1510.

21. Crutzen R, Giabbanelli PJ, Jander A et al. (2015) Identifying binge drinkers based on parenting dimensions and alcohol-specific parenting practices: building classifiers on adolescent-parent paired data. BMC Public Health 15, 747 . 